\title{
Polymorphisms in the interleukin 4 receptor and interleukin 13 genes in immediate allergic reactions to beta-lactam antibiotics: A case-control study
}

\author{
Leila Ksouri, ${ }^{1,2 *}$ Yahia Mouloud, ${ }^{1}$ Nancy Dumais ${ }^{2}$
}

\begin{abstract}
Background: Immediate hypersensitivity reactions to beta-lactams are IgE-mediated and constitute the most common adverse reactions to antibiotics mediated by a specific immunologic mechanism.

Objective: We investigated the association between four functional polymorphisms of IL13 (R130Q variant) and IL4RA (I50V, S478P and Q551R variants) genes and susceptibility to immediate allergic reactions to beta-lactams in the Algerian population.
\end{abstract}

Methods: We determined these gene variants in 199 patients and 99 healthy controls from Algeria. In a case-control study using the TaqMan method, we genotyped four single nucleotide polymorphisms (SNPs) including Arg130Gln in IL13, and Ile50Val, Ser478Pro as well as Gln551Arg in IL4RA.

Results: IL4RA I50V variant was more significantly connected with the risk of beta-lactam allergy $(P=0.0144)$ and the total serum IgE level in patients $(P=0.0136)$. A significant correlation was observed between IL13 R130Q and betalactam allergy $(P=0.0384)$. Also, a significant gene-gene interaction was detected between the predominant allele of the IL13 R130Q polymorphism and the three polymorphisms of IL4RA $(P<0.0001, P=0.0163$, and 0.0301 , respectively). Haplotype analysis of IL4RA revealed that GTA haplotype had a significant correlation in patients with beta-lactam allergy $(P=0.0123)$.

Conclusions: Our results indicate that IL4RA (I50V) and IL13 R130Q are associated with beta-lactam allergy. The combination of IL13 and IL4RA variants markedly increases an individual's susceptibility to beta-lactam allergy in the Algerian population.

Key words: Allergy, Beta-lactam, IgE, Interleukin-13, Interleukin-4 receptor, Polymorphism.

\section{From:}

${ }^{1}$ Laboratoire de Biotechnologie des Molécules Bioactives et de la Physiopathologie Cellulaire, Université de Batna 2, Batna, Algérie.

${ }^{2}$ Département de Biologie, Faculté des Sciences, Université de Sherbrooke, Sherbrooke (QC), Canada.

\section{Introduction}

Allergic reactions to beta-lactams are the most common cause of drug reactions mediated by specific immunological mechanisms, where immunoglobulin E (IgE) and T-cells play a role in the onset of allergic reactions. ${ }^{1}$ Hypersensitivity reactions are classified as either immune-mediated reactions or non-immune mediated reactions. Immediate hypersensitivity reactions are usually induced by an IgE-mediated mechanism and occur within the first hour following the last drug administration.

\footnotetext{
* Corresponding author:

Leila Ksouri

Département de Biologie, Faculté des Sciences,

Université de Sherbrooke, 2500 boul de l'Université, Sherbrooke (QC),

Canada, J1K 2R1

E-mail: leila.ksouri.lechekhab@gmail.com
}

These reactions typically appear as urticaria, angioedema, rhinitis, bronchospasm, or anaphylaxis. ${ }^{2,3}$ However, the mechanism by which allergic reactions are induced by beta-lactam antibiotics remains unclear. ${ }^{4}$

IgE-mediated reactions also called immediate hypersensitivity reactions (Type-I hypersensitivity reactions) are classified as humoral mediated reactions. When exposed for the first time to an immunogenic drug, T-cells specifically T-helper-2 (Th2) 
cells, initiate an allergic reaction by releasing interleukin-4 and interleukin-13 (IL4, IL13), which activate and induce proliferation of B-cells. Then, activated B-lymphocytes produce antigen-specific Ig-E. There is a cross-link between multivalent antigen and basophils or mast cells by Ig-E specific for that antigen which leads to the degranulation of basophils and mast cells and release of inflammatory mediators. ${ }^{5}$ Interleukins secreted by Th2 cells, predominantly IL4 and IL13, are critical cytokines in the pathogenesis of allergic disorders. These interleukins share many biological and biochemical characteristics. ${ }^{6}$ Both IL4 and IL13 use the IL4 receptor a chain (IL4RA) as a component of their receptors and transmit their signals through IL4RA. ${ }^{7}$ Several studies reported in Europe, United States of America (USA), and China have also shown that immediate-type allergic reactions to beta-lactams are influenced by three genes that affect IgE production, IL13, IL4, and IL4 receptor a (IL4RA).$^{8-14}$ In the present study, we thus aimed to evaluate the correlation between IgE-mediated reactions to beta-lactams and polymorphisms of IL13 (R130Q) and IL4RA (I50V, S478P, and Q551R variants) in the Algerian population.

\section{Methods \\ Patients' samples}

Samples were taken from Allergy Unit at the Faculty of Medicine of Batna University in Algeria. The study was performed in 199 Algerian patients with immediate-type reaction to beta -lactams (penicillin or cephalosporins) occurring within 1 hour after drug administration, with positive skin tests and/or serum -specific IgE assays. The 99 healthy controls showed negative skin test to beta-lactam and had no history of allergic, dermatologic, or respiratory diseases, or autoimmune diseases such as asthma, eczema, allergic rhinitis, and urticaria. They have no family relationship with cases. Informed consent was obtained from all subjects and the study was conducted according to the declaration of Helsinki Principles, and the ethics committee of Centre Hospitalo-Universitaire de Batna (CHUB, Algérie) approved the study.

\section{IgE levels measurments and TaqMan method}

Five $\mathrm{mL}$ of blood was taken from each participant under complete aseptic conditions and divided into two portions; $1.5 \mathrm{~mL}$ of whole blood was collected in sterile EDTA-containing tubes for DNA extraction, and the rest was left for 30 to 60 minutes for spontaneous clotting at room temperature and then centrifuged at $3000 \mathrm{rpm}$ for 10 minutes. Serum samples were separated into another set of tubes and kept frozen at $-20^{\circ} \mathrm{C}$ for determination of total IgE. Total serum IgE levels were measured by sandwich enzyme-linked immunosorbent assay ELISA (Innovative research Inc, Novi, Michigan, USA) following the manufacturer's protocol. "Non enzymatic salting out" method was used to isolate genomic DNA from peripheral blood. ${ }^{15}$ All the polymorphisms were genotyped by allelic discrimination polymerase chain reaction assays (5' nuclease assay) using predesigned TaqMan SNP Genotyping Assays (Applied Biosystems, USA). Both PCR primers and MGB TaqMan probes are shown in Table 1. Primers and probes annealing temperatures for all allele-discriminating assays were optimized using a standard PCR setup on a Bio-Rad CFX connect real-time PCR instrument (Bio-Rad Laboratories, Hercules, CA, USA). The program consisted of 3 minutes of polymerase activation at $98^{\circ} \mathrm{C}$, followed by 40 cycles of collective annealing and elongation steps at $52-64^{\circ} \mathrm{C}$ (temperature gradient) for 30 seconds, and denaturation at $98^{\circ} \mathrm{C}$ for 15 seconds. For the optimization of the primer concentration, a titration series of each pair was prepared from 200 to $600 \mathrm{nM}$, with $300 \mathrm{nM}$ of each of the two probes added, and using a heterozygotic sample as template DNA. Optimal annealing temperature, concentrations of primers and probes were selected based on the efficiency of the real-time PCR amplification. The main advantages of the direct approach for genotyping are less hands -on time during setup, and that the PCR is performed in a closed system, hereby minimizing the risk of contamination.

Reactions were performed in a $12 \mu \mathrm{L}$ volume, consisting of six $\mu \mathrm{L}$ Bio-Rad SsoAdvenced Universal Probes Supermix, 500 nM of unlabeled PCR primers, $300 \mathrm{nM}$ of TaqMan MGB probes, and $10 \mathrm{ng}$ of template DNA. Thermal cycling was initiated with a denaturation step of $3 \mathrm{~min}$ at $98^{\circ} \mathrm{C}$, followed by 40 cycles of $15 \mathrm{~s}$ at $98^{\circ} \mathrm{C}$ and $30 \mathrm{~s}$ at $60^{\circ} \mathrm{C}$. After PCR were completed, allelic discrimination was analyzed using the Bio-Rad CFX Manager Software (Version 3.1, Bio-Rad). Genotype assignment was determined by plotting the endpoint relative fluorescent units (RFU) for one fluorophore (allele one on the $\mathrm{x}$-axis) against the RFU for the other fluorophore (allele two on the y-axis) on the allelic discrimination plot. All samples were set up in triplicate. PCR reactions were performed in a dedicated PCR area with dedicated PCR pipettes and reagents. For quality control purposes, each real time-PCR included negative as well as positive controls for all the genotypes. For validation, about $10 \%$ of the

Table 1. Primers and probes for genotyping screening by TaqMan allelic discrimination.

\begin{tabular}{|c|c|c|c|c|}
\hline SNP & $\begin{array}{l}\text { NCBI rs } \\
\text { No }\end{array}$ & $\begin{array}{l}\text { Base } \\
\text { change }\end{array}$ & Primers & Probes \\
\hline IL13 Arg130Gln & rs20541 & $\mathrm{G}>\mathrm{A}$ & $\begin{array}{l}\text { F: 5'-CTGCAAATAATGATGCTTTCGA-3' } \\
\text { R: 5'-CCAGTTTGTAAAGGACCTGCTCT-3' }\end{array}$ & $\begin{array}{l}\text { A allele: 5'-FAM-GAGGGACAGTTCAACTG-MGB-3' } \\
\text { G allele: 5'-HEX-GAGGGACGGTTCAACT-MGB-3' }\end{array}$ \\
\hline IL4RA Ile50Val & rs1805010 & $A>G$ & $\begin{array}{l}\text { F: 5'-CTACAGGTGACCAGCCTAAC-3' } \\
\text { R: 5'-CCCACAGGTCCAGTGTATAGT-3' }\end{array}$ & $\begin{array}{l}\text { G allele: 5'-FAM-ACGTGTGTCCCTG-MGB-3' } \\
\text { A allele: 5'-HEX-ACGTGTATCCCTG-MGB-3' }\end{array}$ \\
\hline IL4RA Ser478Pro & rs1805015 & $\mathrm{T}>\mathrm{C}$ & $\begin{array}{l}\text { F: 5'-CGCAGGCAACCCTGCTTA-3' } \\
\text { R:5'-GCATCTCGGGTTCTACTTCCTC-3' }\end{array}$ & $\begin{array}{l}\text { C allele: 5'-FAM-CAGCAACCCCCTGAG-MGB-3' } \\
\text { T allele: 5'-HEX-TTCAGCAACTCCCTGAG-MGB-3' }\end{array}$ \\
\hline IL4RA Gln551Arg & rs1801275 & $A>G$ & $\begin{array}{l}\text { F: 5'-CTCCGCCGAAATGTCCTC-3' } \\
\text { R: 5'-GCCTTGTAACCAGCCTCTCC-3' }\end{array}$ & $\begin{array}{l}\text { G allele: 5'-FAM-GGCTATCGGGAGTTT-MGB-3' } \\
\text { A allele: 5'-HEX-TGGCTATCAGGAGTTTG-MGB-3' }\end{array}$ \\
\hline
\end{tabular}


samples were re-genotyped. The results were reproducible with no discrepancies in genotyping.

\section{Statistical analysis:}

We used SNPstats software to test Hardy-Weinberg (HW) equilibrium of alleles frequencies. ${ }^{16}$

This software was also used to estimate haplotype frequencies in cases and controls. The chi-square test was used to test for significant association between beta-lactam allergies and alleles or genotypes. Odds ratio (OR), used as a measure of association strength, and the corresponding 95\% confidence interval (CI) was calculated. Kruskal-Wallis test was used to assess whether the distribution of a categorical variable is the same between genotype groups. A $P$-value of less than 0.05 was considered significant. Statistical analyses were performed using GraphPad Prism version 7 (GraphPad Software, San Diego, CA).

\section{Results}

In the present case-control study, we explored the association between the IL13, IL4RA polymorphisms and beta-lactam allergy in a sample of Algerian population. The association between the immediate allergic reaction to beta-lactams and polymorphisms of IL13 (R130Q), IL4RA (I50V, S478P and Q551R) was evaluated in 199 patient and 99 healthy controls from Algeria. There were no significant differences in the distribution of age $(P=0.1023)$ and sex $(P=0.5554)$ between the cases and controls (Table 2). Patients with immediate allergic reactions had a significantly higher concentration of total serum IgE than controls (Table 2). All genotyped distributions of control subjects were consistent with those expected from the Hardy-Weinberg equilibrium $(\mathrm{P}>0.05)$. Besides, the minor allele frequency (MAF) of all the four SNPs was consistent with that reported in the HapMap database (Table 3). No linkage disequilibrium was found between IL13 and IL4RA polymorphisms.

Genotype distributions and allele frequencies of all analyzed polymorphisms for the patients and control group are shown in Table 2. The frequency of the predominant alleles of IL4RA I50V and IL13 R130Q was significantly higher in patients than in controls, whereas no difference was observed for the IL4RA S478P and IL4RA Q551R (Table 2). We observed a significant association between IL13 R130Q and total serum level of IgE

Table 2. Clinical characteristics and genotypes and allele frequencies of IL13 and IL4RA of patients and controls.

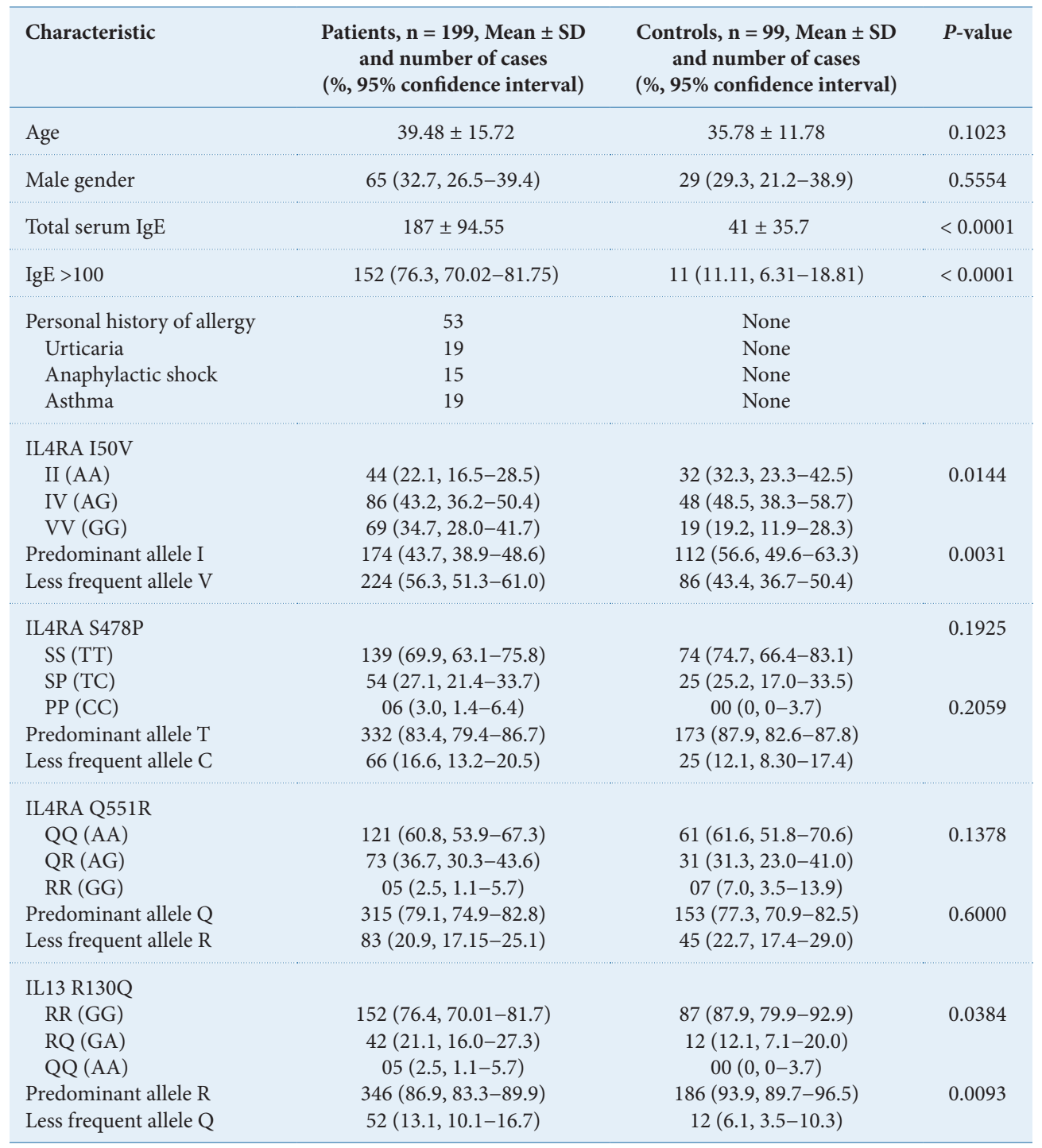


Table 3. Primary information of genotyped SNPs in the IL13 and IL4RA genes.

\begin{tabular}{|c|c|c|c|c|c|c|c|}
\hline \multirow[t]{2}{*}{ SNP } & \multirow[t]{2}{*}{ NCBI rs No } & \multirow[t]{2}{*}{ Location } & \multirow[t]{2}{*}{ Base change } & \multicolumn{3}{|c|}{ MAF } & \multirow[t]{2}{*}{$P$ for $\mathrm{HWE}^{\mathrm{b}}$} \\
\hline & & & & HapMap $^{a}$ & Case & Control & \\
\hline IL13 Arg130Gln & rs 20541 & exon 4 & $\mathrm{G}>\mathrm{A}$ & 0,130 & 0.13 & 0.07 & 0,991 \\
\hline IL4RA Ile50Val & rs 1805010 & exon 5 & $A>G$ & 0,425 & 0.51 & 0.43 & 0,990 \\
\hline IL4RA Ser478Pro & rs1805015 & exon 12 & $\mathrm{~T}>\mathrm{C}$ & 0,152 & 0.17 & 0.13 & 0,350 \\
\hline IL4RA Gln551Arg & rs1801275 & exon 12 & $A>G$ & 0,207 & 0.21 & 0.23 & 0,260 \\
\hline
\end{tabular}

a MAF from the HapMap database

${ }^{\mathrm{b}} \mathrm{HWE} \mathrm{P}$ value in the control group

in patients as well as controls $(P=0.0002)$. The association of IL4RA I50V and S478P with total IgE was more significant when restricting the analysis to patients (Table 4).

Because of the biological relationship of IL4RA and IL13, an analysis was performed to determine if individuals with the risk genotypes for both genes were at higher risk of developing beta-lactam allergy. The data are summarized in Figure $\mathbf{1}$ and showed that IL13 130RR combined with any of the predominant homozygous genotypes of IL4RA was a risk factor in allergy to beta-lactams. A similar analysis was performed examining total serum IgE levels. Our results showed IL13/IL4RA variant combination: $P=0.0220,0.0002,0.0020$, respectively and each variant $P=0.00020 .2224,0.6978,0.1237$, respectively. A linkage disequilibrium (LD) analysis was performed to study the relationships between the three SNPs of IL4RA and beta -lactam allergy. The LD showed that rs1805010 and rs1805015 had linkage disequilibrium with $\mathrm{D}^{\prime}$ of 0.5195 , rs1805015 and rs 1801275 had a score of $D^{\prime}=0.7977$. However, rs1805010 and rs1801275 did not show linkage disequilibrium. Three haplotypes were found in the three SNPs of IL4RA gene: ATA, GTA, and GCG (Table 5). These haplotypes were observed in the case and control groups $(P<0.0001, P=0.0123$, and 0.3099 , respectively). The haplotype GTA is correlated with beta-lactam allergy in Algerian population. Indeed, the haplotype GTA was significantly more frequent in patients with immediate allergic reactions to beta-lactams than in control subjects $(P=$ $0.0123)$. Interestingly, the haplotype ATA was significantly more frequent in controls subjects than in patients $(P<0.0001)$.

Table 4. Serum total IgE levels in patients with beta-lactam allergy.

\begin{tabular}{lcc}
\hline \multirow{2}{*}{ Polymorphism } & Total IgE $(\mathbf{I U} / \mathbf{m l})$ & \multirow{2}{*}{$\boldsymbol{P}$-value } \\
\cline { 2 - 3 } & Median $\left(\mathbf{2 5}^{\text {th }}-\mathbf{7 5}^{\text {th }}\right)$ & \\
\hline IL4RA I50V & $168.3(81.03-253)$ & \\
II & $216.5(82.75-259.6)$ & \\
IV & $252.4(181.6-269.1)$ & \\
VV & & 0.0492 \\
IL4RA S478P & $218.3(100.5-276)$ & \\
SS & $197.4(97.78-258.5)$ & 0.2011 \\
SP & $261.6(260.7-264.2)$ & \\
PP & & \\
IL4RA Q551R & $219.2(110.7-273.1)$ & \\
QQ & $213.4(90.75-261.6)$ & \\
QR & $112(63.3-180.9)$ & \\
RR & & \\
IL13 R130Q & $214.4(92.38-261.1)$ & \\
RR & $213.7(146.6-277.5)$ & \\
RQ & $270.2(240-301)$ & \\
QQ & & \\
\hline
\end{tabular}

Table 5. Major haplotype frequencies of IL4RA in the case and control groups.

\begin{tabular}{llcccc}
\hline \multirow{2}{*}{ Genotype } & \multirow{2}{*}{ Haplotype } & \multicolumn{2}{c}{ Frequency } & \multirow{2}{*}{$\boldsymbol{P}$-value } & OR (95\% CI) \\
\cline { 3 - 4 } & & \multicolumn{2}{c}{ Case } & Control & \\
\hline IL4RA & & & & & \\
rs1805010 & ATA & $150(0.378 \%)$ & $96(0.486 \%)$ & $<0.0001$ & $0.45(0.31-0.65)$ \\
rs1805015 & GTA & $152(0.383 \%)$ & $54(0.275 \%)$ & 0.0123 & $1.61(1.11-2.35)$ \\
rs1801275 & GCG & $95(0.240 \%)$ & $45(0.230 \%)$ & 0.3099 & $1.23(0.82-1.83)$ \\
\hline
\end{tabular}



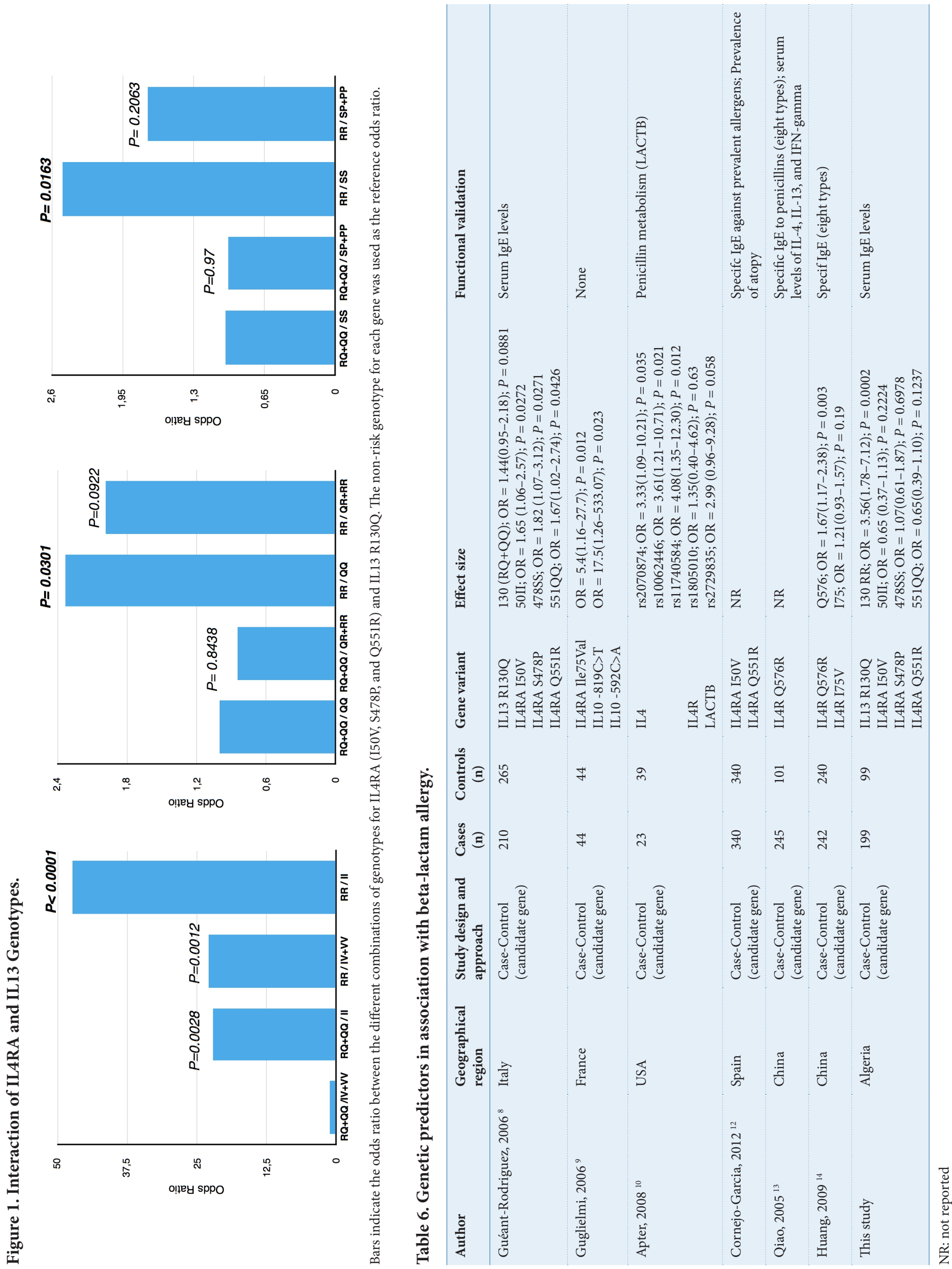


\section{Discussion}

Several studies suggested that allergic reaction to beta-lactams are influenced by genes involved in IgE production, including IL13 and IL4 pathways. ${ }^{8-14,17,18}$ Besides, recent population studies have reported an association between IL13 and IL4RA with atopy and asthma. ${ }^{19-21}$ In this study, we found for the first time in the Algerian population, an association of rs 1805010 polymorphism in IL4RA gene and rs20541 in IL13 with an allergic reaction to beta-lactams.

In Algerian patients with allergic reaction to beta-lactams, we observed a higher concentration of total serum IgE than non-allergic patients suggesting the involvement of a genetic mechanism related to IgE class switching. Supporting our data, a relationship was found among IL4RA I50V and IL13 R130Q polymorphisms, the risk of immediate reaction to beta-lactams, and total serum IgE level. ${ }^{8}$ However, Apter et al. reported that the IL4RA I50V polymorphism had no relationship with penicillin allergy based on a series of 23 self-reported penicillin -allergic patients from USA. ${ }^{10}$ One possible explanation for this discrepancy is the difference in the genotype frequency of ILR4A I50V between different populations. This explanation is supported by the research of Gueant et al. who showed that the IL4RA I50V of the AA genotype was more significantly associated with the risk of penicillin allergy than with the risk of cephalosporin allergy ${ }^{17}$ This study also demonstrated that a difference in the AA genotype frequency of IL4RA I50V existed between two Europeans populations. ${ }^{17}$

The IL4RA gene is located on chromosome 16p11-16p12. It is a subunit that plays a key role in allergic disease by promoting the IgE production..$^{22}$ In our study, the I50V and S478R were correlated with $\operatorname{IgE}$ production in patients, whereas the Q551R was not associated with the IgE level (Table 4). However, Cornejo-Garcia et al. found that total IgE was affected by Q551R polymorphism as well as IL13 130RQ/QQ and IL4RA 551QQ epistatic genotype in Spanish Caucasians. ${ }^{12}$ In our series, the two symmetrical combinations (IL13 130RR and IL4RA 50II, IL13 130RR and IL4RA 551QQ) are significantly correlated with total IgE level, but less than the effect of IL13 R130Q alone $(P=0.0002)$, confirming the critical role of IL13 in the initiation of IgE production. ${ }^{23-26}$ These gene-gene interactions were consistent with the complementary role of both molecules in IgE switching. ${ }^{8}$ Another interesting finding of our study, is the combination of the predominant allele of IL13 R130Q polymorphism with any of the predominant homozygous genotypes of the three polymorphisms of IL4RA (I50V, S478P, and Q551R) was more significantly associated with the risk of beta-lactam allergy $(P<0.0001, p=0.0163,0.0301$, respectively $)$ than any polymorphism considered alone $(P=0.0093,0.0031$, $0.2059,0.6000$, respectively). Also, the symmetrical combinations (IL13 130RQ/QQ and IL4RA 50II), and (IL13 130RR and IL4RA 50IV/VV) were significantly associated with the risk of beta-lactam allergy, while the other combinations were not significant (Figure 1). Table 6 shows genetic association studies that reported genetic predictors in association with beta-lactam allergy compared with our study. These studies suggested that pro-inflammatory cytokine genes such as IL4R, IL4, IL13 are involved in IgE mediated beta-lactam reactions.
Computer modelling of the rs20541 variant has shown that this substitution affects the signal strength between interleukin 13 and its receptor. ${ }^{27}$ This polymorphism encodes an amino acid residue, which is located within the D helix, close to the C-terminal region of IL13. ${ }^{28}$ IL13 is a ligand of the IL4RA subunit; it is thus possible that the R130Q polymorphism influences the interaction between D helix and the IL4RA subunit. The underlying molecular mechanisms of this association need to be clarified because the computer modelling of the IL13/ IL4RA interaction suggests that the arginine of the 130RR variant repulses the histidine 131 of IL4RA. ${ }^{27}$ The S478P and Q551R variants of IL4RA may intensify the downstream signalling, because of their position close to a STAT6-recruiting domain. ${ }^{28}$ Therefore, additional genes related to the signalling pathways of IL4RA, such as IL4, STAT6, and JAK1, could also account for an additional risk of IgE mediated allergy to beta-lactams, as previously suggested in probands with asthma susceptibility. ${ }^{21}$

In the haplotype analysis of the IL4RA gene, the GTA haplotype frequency in patients with beta-lactam allergy was found to be significantly higher than the control group suggesting an interaction between the three polymorphisms regarding susceptibility to beta-lactam allergy. In other words, the results indicate that GTA haplotype could be associated with the susceptibility to beta-lactam allergy in the Algerian population. The association of G50, T478 and A551 combination with beta-lactam allergy was higher than each allele alone, suggesting that haplotype analysis can provide more information than the single SNP alone. Moreover, it is interesting to observe that the haplotype ATA seems to have a protective effect against beta-lactam allergy, although the reason is unclear. Thus further studies should be undertaken to analyse the putative relevance of haplotypes of IL4RA Ile50Val, Ser478Pro and Gln551Arg polymorphisms in the development of beta-lactam allergy.

\section{Conclusion}

In summary, our study suggests that IL4RA I50V and IL13 R130Q polymorphisms are related to beta-lactam allergy. Our data demonstrate that IL13 is a more potent predictor of beta-lactam allergy than IL4RA. In the Algerian population, a significant association of IL13/IL4RA polymorphism combinations with beta-lactam allergy and IgE levels is observed. However, additional studies are needed to confirm these results in other populations. Also, our data suggest that the haplotype GTA from rs1805010, rs1805015, and rs1801275 of IL4RA may be related somehow to beta-lactam allergy. This relationship needs to be further studied using a larger sample.

Our results have a certain clinical implication. The identification of genetic risk factors may improve the diagnosis and understanding of the pathophysiology of beta-lactam allergy. Therefore, having a clear view of the genetic factors involved can lead us to develop better preventive methods and strategies as well as effecting better drug design and treatment strategies in the future. 


\section{Conflicts of Interests}

The authors have not declared any conflict of interests

\section{Acknowledgements}

This study was supported in part by the Natural Sciences and Engineering Research Council of Canada (Funding Reference Number RGPIN-2015-06306), and by Biotechnology Laboratory of the Bioactive Molecules and the Cellular Physiopathology, University of Batna 2, Algeria.

\section{References}

1. Mahmoud KH, Alzolibani AA, Rasheed Z, Farouk Y, Saif GB, Al Robaee AA. Interleukin- 4 and interferon- $\gamma$ are possible allergic markers in pediatric patients with $\beta$-lactam hypersensitivity. Int J Appl Basic Med Res. 2016;6: 276-81.

2. Blanca M, Cornejo-Garcia JA, Torres MJ, Mayorga C. Specificities of B cell reactions to drugs. The penicillin model. Toxicology. 2005;209:181-4

3. Bousquet PJ, Kvedariene V, Co-Minh HB, Martins P, Rongier M, Arnoux B, et al. Clinical presentation and time course in hypersensitivity reactions to beta-lactams. Allergy. 2007;62:872-6.

4. Comte D, Petitpierre S, Bart PA, Spertini F. Allergie aux $\beta$-lactamines. Rev Med Suisse. 2012;8:836-42.

5. DiPiro JT, Ownby DR, Schlesselman LS. Allergic and Pseudoallergic Drug Reactions. In: DiPiro JT, Talbert RL, Yee GC, Matzke GR, Wells BG, Posey LM, editors. Pharmacotherapy, a pathophysiologic approach. New York: McGraw-Hill, 2002. p. 1585-96.

6. Callard RE, Matthews DJ, Hibbert L. IL-4 and IL-13 receptors: are they one and the same? Immunol Today. 1996;17:108-10.

7. Hershey GK. IL-13 receptors and signaling pathways: an evolving web. J Allergy Clin Immunol. 2003;111:677-90.

8. Gueant-Rodriguez RM, Romano A, Beri-Dexheimer M, Viola M, Gaeta F, Gueant JL. Gene-gene interactions of IL13 and IL4RA variants in immediate allergic reactions to betalactam antibiotics. Pharmacogenet Genomics. 2006;16:713-9.

9. Guglielmi L, Fontaine C, Gougat C, Avinens O, Eliaou JF, Guglielmi P, et al. IL-10 promoter and IL-4R alpha gene SNPs are associated with immediate beta-lactam allergy in atopic women. Allergy. 2006;61:921-7.

10. Apter AJ, Schelleman H, Walker A, Addya K, Rebbeck T. Clinical and genetic risk factors of self-reported penicillin allergy. J Allergy Clin Immunol. 2008;122:152-8.

11. Gueant JL, Gueant-Rodriguez RM, Aimone Gastin I, Cornejo-Garcia A, Viola M, Barbaud A, et al. Pharmacogenetic determinants of immediate and delayed reactions of drug hypersensitivity. Curr Pharm Des. 2008;14: 2770-7.

12. Cornejo-Garcia JA, Gueant-Rodriguez RM, Torres MJ, Blanca-Lopez N, Tramoy D, Romano A, et al. Biological and genetic determinants of atopy are predictors of immediate-type allergy to betalactams, in Spain. Allergy. 2012;67:1181-5.
13. Qiao HL, Yang J, Zhang YW. Relationships between specific serum IgE, cytokines and polymorphisms in the IL-4, IL-4R alpha in patients with penicillin allergy. Allergy. 2005;60:1053-9.

14. Huang CZ, Yang J, Qiao HL, Jia LJ. Polymorphisms and haplotype analysis of IL-4R alpha Q576R and I75V in patients with penicillin allergy. Eur J Clin Pharmacol. 2009;65:895-902.

15. Suguna S, Nandal DH, Kamble S, Bharatha A, Kunkulol R. Genomic DNA isolation from human whole blood samples by non enzymatic salting out method. Int J Pharm Pharm Sci. 2014;6:198-9.

16. Sole X, Guino E, Valls J, Iniesta R, Moreno V. SNPStats: a web tool for the analysis of association studies. Bioinformatics. 2006;22:1928-9.

17. Gueant JL, Gueant-Rodriguez RM, Cornejo-Garcia JA, Viola M, Blanca M, Romano A. Gene variants of IL13, IL4, and IL4RA are predictors of beta-lactam allergy. J Allergy Clin Immunol. 2009;123:509; author replay 509-10.

18. Li J, Liu XY, Li LJ, You C, Shi L, Zhang S, et al. Correlation analysis of gene polymorphisms and $\beta$-lactam allergy. J of Zhejiang Univ-Sci B (Biomed \& Biotechnol). 2015;16:632-9.

19. Narozna B, Hoffmann A, Sobkowiak P, Schoneich N, Breborowicz A Szczepankiewicz A. Polymorphisms in the interleukin 4, interleukin 4 receptor and interleukin 13 genes and allergic phenotype: A case control study. Adv Med Sci. 2016;61:40-5.

20. Li J, Lin LH, Wang J, Peng X, Dai HR, Xiao H, et al. Interleukin-4 and interleukin-13 pathway genetics affect disease susceptibility, serum immunoglobulin E levels, and gene expression in asthma. Ann Allergy Asthma Immunol. 2014;113:173-9.

21. Howard TD, Koppelman GH, Xu J, Zheng SL, Postma DS, Meyers DA et al. Gene-gene interaction in asthma: IL4RA and IL13 in a Dutch population with asthma. Am J Hum Genet. 2002;70:230-6.

22. Wu LC, Scheerens H. Targeting IgE production in mice and humans. Curr Opin Immunol. 2014;31:8-15.

23. Punnonen J, Yssel H, Vries JE. The relative contribution of IL- 4 and IL-13 to human IgE synthesis induced by activated CD4+ or CD8+ T cells. J Allergy Clin Immunol. 1997;100:792-801.

24. de Waal Malefyt R, Abrams JS, Zurawski SM, Lecron JC, Mohan -Peterson S, Sanjanwala B, et al. Differential regulation of IL-13 and IL-4 production by human CD $8+$ and CD $4+$ Th0, Th1 and Th2 T cell clones and EBV-transformed B cells. Int Immunol. 1995;7:1405-16.

25. de Vries JE. The role of IL-13 and its receptor in allergy and inflammatory responses. J Allergy Clin Immunol. 1998;102:165-9.

26. Choi WA, Kang MJ, Kim YJ, Seo JH, Kim HY, Kwon JW, et al. Gene-gene interactions between candidate gene polymorphisms are associated with total IgE levels in Korean children with asthma. J Asthma. 2012;49:243-52.

27. Heinzmann A, Mao XQ, Akaiwa M, Kreomer RT, Gao PS, Ohshima K, et al. Genetic variants of IL-13 signalling and human asthma and atopy. Hum Mol Genet. 2000;9:549-59.

28. Zurawski SM, Vega F Jr, Huyghe B, Zurawski G. Receptors for interleukin -13 and interleukin- 4 are complex and share a novel component that functions in signal transduction. EMBO J. 1993;12:2663-70. 